\title{
Human flexor reflexes
}

\author{
BHAGWAN T. SHAHANI ${ }^{1}$ AND ROBERT R. YOUNG ${ }^{2}$
}

From the University Department of Neurology, The Churchill Hospital, Oxford

SUMMARY One type of flexor reflex, that recorded from the tibialis anterior muscle in response $\overrightarrow{0}$ to electrical stimulation of the sole of the foot, was studied in normal subjects and patients with several neurological disorders. Normally this reflex consists of two components, the second of which $\frac{\overline{\bar{\rho}}}{\bar{\nabla}}$ is related to the actual withdrawal. The first component, normally of lower threshold, is difficult to $\stackrel{\mathbb{Q}}{\square}$ evoke in patients with chronic spinal cord or discrete cerebral lesions, whereas it has an unusually low threshold and is very clearly seen in those with Parkinson's disease. In patients with spinal cord $\vec{\circ}$

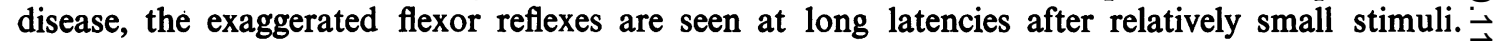
During the early phase of recovery from spinal transection, both components may be seen and are, $\vec{\omega}$ therefore, spinal in origin. Studies of patients with the sensory neuropathy of Friedreich's ataxia suggest that the afferent fibres responsible for these flexor reflexes are the small myelinated fibres. Recovery curves demonstrate very long-lasting changes in flexor reflex excitability in normal subjects $\underset{+}{\omega}$ and patients with 'spasticity' from spinal lesions. This differs in patients with 'spasticity' from lesions ir rostral to the brain-stem. Examples in man of such physiological phenomena as reciprocal inhibitio $\overrightarrow{m_{7}} \frac{\vec{\sigma}}{\sigma}$ local sign, habituation, temporal and spatial summation are discussed.

Flexor reflexes have been of interest to physiologists at least since the early part of this century, and Sherrington (1910) and his collaborators (Creed, Denny-Brown, Eccles, Liddell, and Sherrington, 1932) were pre-eminent in their study. A number of detailed investigations of human flexor reflexes have been carried out in the past 20 years (Kugelberg, 1948, Pedersen, 1954, Hagbarth, 1960, Kugelberg, Eklund, and Grimby, 1960, Hagbarth and Finer, 1963, Dimitrijević and Nathan, 1968), but because of multiple variations in technique, it is often very difficult to compare the results obtained by different investigators.

We began this study of flexor reflexes in an effort to understand something of human spinal cord function and the way this is changed pathologically to produce such abnormalities as flexor spasms (Shahani and Young, 1971), 'spasticity' and 'rigidity'. Because of the enormous complexity introduced by the very large number of possible combinations and permutations of stimulus site, muscles to be recorded from, and positions and background activity im-

'Supported by Association for the aid of Crippled Children, New York. 'Supported in part by a Special Fellowship (2F11NB1539-02) from The National Institute of Neurological Disease and Blindness.

1,2Present address: Massachusetts General Hospital, Boston, Massachuset ts 02114, U.S.A. posed upon these muscles before stimulation, it wogs obvious that we would have to simplify our teci niques in some way. A series of pilot experimens was therefore undertaken to discover which of te experimental methods would be best for our.purposes.

We sought methods which were as simple, painless, and natural or physiological as possible and which could be applied to subjects who were quadriplegic as well as to those who were normal or $\stackrel{\square}{\square}$ nearly so. Results which were reproducible and free $\overrightarrow{\vec{F}}$ from as many of the uncontrolled variables attend-응 ant upon human investigation as possible were essential. A brief report of some of the normal findings has already appeared (Shahani and Young, 1969).

\section{METHODS}

We have studied five normal male subjects, aged 24 to $ᄋ$ 49 , who were without history or signs of neurological disease, and 25 neurological patients. The paretic leg 음 was studied in five males, aged 42 to 59 , who had localiz- $>$ able old cerebral lesions-four had discrete missile wounds from the second world war and one a lateral putamenal haemorrhage evacuated through the parietal $N$ cortex. Five males, aged 44 to 69, with Parkinson's $N$ disease were studied. The remaining 15 had evidence of $\bigcirc$ bilateral spinal cord disease. Two males, 42 and 63 years $\omega$ 
old, had longstanding, moderate spastic parapareses with thoracic and cervical cord compression, respectively. One 49 year old man had a complete traumatic cord transection at $\mathrm{C} 6$ segment. He was studied from the stage of spinal shock for the next three months. Eight patients were considered to have multiple sclerosis with bilateral spinal lesions. Of these, one male and four females, aged 28 to 51 , did not have symptomatic flexor spasms though they could easily be elicited on examination. Two males and one female, 46 to 55 years of age, complained of frequent, severe, and painful spontaneous flexor spasms. The remaining four patients, 44 to 59 years old, had far-advanced Friedreich's ataxia, and all complained of mild spontaneous flexor spasms on questioning. The authors were studied repeatedly, and the other subjects and patients were studied on one to eight occasions on different days, the mean being three sessions per person. No medication or sedation was given in connection with these studies; the subjects lay comfortably supine in a warm room and were awake. Stimulation, except as noted, was carried out electrically, and the stimuli were led through an isolation transformer from a DISA Multistim stimulator. Stimuli were triggered without warning in a random fashion, using a silent pushbutton which was not visible to the subject. Electromyographic activity was recorded with electrodes which were connected by a cathode follower input through Tektronix type 122 preamplifiers to separate beams of a cathode ray oscilloscope which were filmed on moving paper. Often six to 10 successive traces were superimposed photographically allowing for estimations of reproducibility. The illustrations depicted here, however, were chosen for their clarity from photographs of single sweeps. Movements could be signalled by feeding the output of a ceramic transducer attached, for instance, to the foot directly into one channel of the oscilloscope. In addition, the muscles and movements produced were palpated and observed. The loudspeaker of the electromyograph was always turned off.

Though mechanical stimulation (pinch or pinprick) is more natural, it is with the use of electrical stimulation that one is able to record the most accurate latency. Furthermore, variations in reflex latency should not be a function of slight changes in stimulus intensity as would be the case if one were working on a steep portion of the strength-latency curve. Stimuli, therefore, should be capable of being made supramaximal. That is, when successive increments in the intensity of a single stimulus or train of stimuli no longer produce a shortening of the response latency, and the stimulus is then increased by a further $25 \%$, it is defined as supramaximal. Whereas a single stimulus would usually produce a flexor reflex, it was rare that a single stimulus could be made supramaximal. Trains of stimuli were used, therefore, and a train of $0.1 \mathrm{msec}$ square waves at a frequency of $500 / \mathrm{sec}$ which lasted 20 msec could be made supramaximal in every case.

Stimulation of the medial sole of the foot at the apex of the plantar arch produced flexor reflexes with minimum thresholds and latencies, when compared with stimulation anywhere else on the lower extremities. The genital and lower sacral areas were never stimulated. Even when using trains of stimuli, one could not always stimulate supramaximally by applying the electrodes to the surface of the skin. It was, therefore, necessary in most instances to stimulate via intradermal electrodes. These were two steel needles, bared at the tips, which were inserted longitudinally $0.5 \mathrm{~cm}$ apart intradermally into the medial sole of the foot at the apex of the plantar arch. The needles protruded from a Perspex block to which they were cemented and which, itself, was securely taped to the foot. Concentric needle recording from adjacent muscles, such as abductor hallucis, gave no evidence that the intradermal stimuli excited muscle directly. Stimulus threshold values from day to day cannot be compared precisely because neither stimulus current nor electrode impedance was measured. Intensity ranged, however, from stimulator settings of 5 to $50 \mathrm{~V}$ and isolation transformer settings of $\times 1$ to 5 . The effectiveness of the stimulus was roughly proportional to both duration and voltage, but changes in voltage were more effective in producing continuous variations in reflex size and latency. Flexor, or withdrawal, responses could easily be set off by the sound of the trigger being pressed or the sound of the stimulus in the loudspeaker. It was, therefore, necessary to have the loudspeaker turned off at all times and to use a special silent pushbutton. Furthermore, the subject could not be allowed to trigger his own stimuli because, in doing so, anticipatory muscular contractions were often seen and characteristic changes in reflex configuration were produced (v.i.). For similar reasons the stimuli could not be delivered in a regular or repeatable fashion. Delays of 45 to 60 seconds between individual stimuli were necessary to allow recovery from one stimulus to the next. Except for the determination of excitability curves, in which case two stimuli were given at shorter intervals, at least 60 seconds elapsed between successive stimuli which were triggered completely unexpectedly as far as the subjects were concerned.

The subjects lay supine with their legs always fully extended and adducted. No background muscle contraction was present in the legs during these studies. The reflex contractions produced were isotonic insofar as there was no hindrance, other than gravity, to movement of the leg. Following each movement, the leg was repositioned in full extension with the ankle as near a $90^{\circ}$ angle as possible.

Electromyographic recording was carried out before, during, and after stimulation both from the surface and by intramuscular needle electrodes. The latter allow more precise localization of the response, but are painful, both when inserted through the skin into the muscle and when the muscle contracts. Pain becomes an experimental variable, affecting reflex patterns as well as general levels of apprehension and should be minimized. Recording was therefore carried out by small, steel, spring-loaded, clip-on electrodes (Copland and Davies, 1964), with the sharpened tips placed superficially into the skin over the muscle. Application of these electrodes is practically painless and when they are securely taped to the skin no pain is produced by movements of the legs. Using them, we hoped to sample as much of the total response as possible. The corresponding disadvantage is that they record activity from muscles at a 
distance. From the gastrocnemius electrodes, we did record activity which was produced in $\mathrm{m}$. tibialis anterior (TA), but the difference in amplitude of the activity produced by voluntary contraction in one muscle, as recorded from the two different sites varied between 12 to 1 and 15 to 1 . More importantly, our preliminary experiments showed no significant difference between surface and intramuscular recording as regards latency and size of the reflex response. These preliminary experiments revealed that, using our technique of stimulation, the reflex response with minimal latency was recorded from TA. Our survey included the femoral flexors as well as other thigh, leg, and intrinsic foot muscles. In some instances the minimal reflex latency in TA was the same as that in one or more of the other muscles, but we could never record a reflex in any of the other muscles with latency shorter than that recorded from TA. The threshold for response in TA was also as low, or lower, than that for any other muscle in the leg, and on occasion, only TA appeared to respond. This muscle is easily localized on the surface and, to minimize the variability of results, electrodes were placed 4 to $5 \mathrm{~cm}$ apart longitudinally over its belly. The primary antagonists to TA are triceps surae and, in particular, soleus muscles. When recording from the surface over the soleus muscle the electrodes are very near to TA, so we routinely placed the other pair of electrodes $4 \mathrm{~cm}$ apart transversely over the two bellies of the gastrocnemius muscle.

\section{RESULTS}

Though a number of studies were done varying both resting posture and background muscle contraction, as well as using more natural mechanical stimulation of different cutaneous areas, we plan primarily to report results from simpler studies in which these other variables remained as constant as possible from one recording session to another. Unless specified, the reflexes mentioned were recorded from TA in response to supramaximal intradermal stimulation of the medial sole of the ipsilateral foot.

NORMAL SUBJECTS Two components were regularly seen making up the flexor reflex, the first with a lower threshold than the second. Concentric needle recordings showed they both arose from TA and often from the same motor units (Shahani, 1968, 1969). The latency of the first component varied between 50 and $60 \mathrm{msec}$ from subject to subject, whereas the second component might begin at a latency of 110 to $400+$ msec depending on stimulus strength. As seen in Fig. 1, increases in stimulus strength resulted in decreases in the latency of the components, though the second shortened over a much wider range than the first. The latter usually decreased by 10 to $20 \mathrm{msec}$ or less. Increasing stimulation also produced increasing amplitude and duration of the components so that, at higher

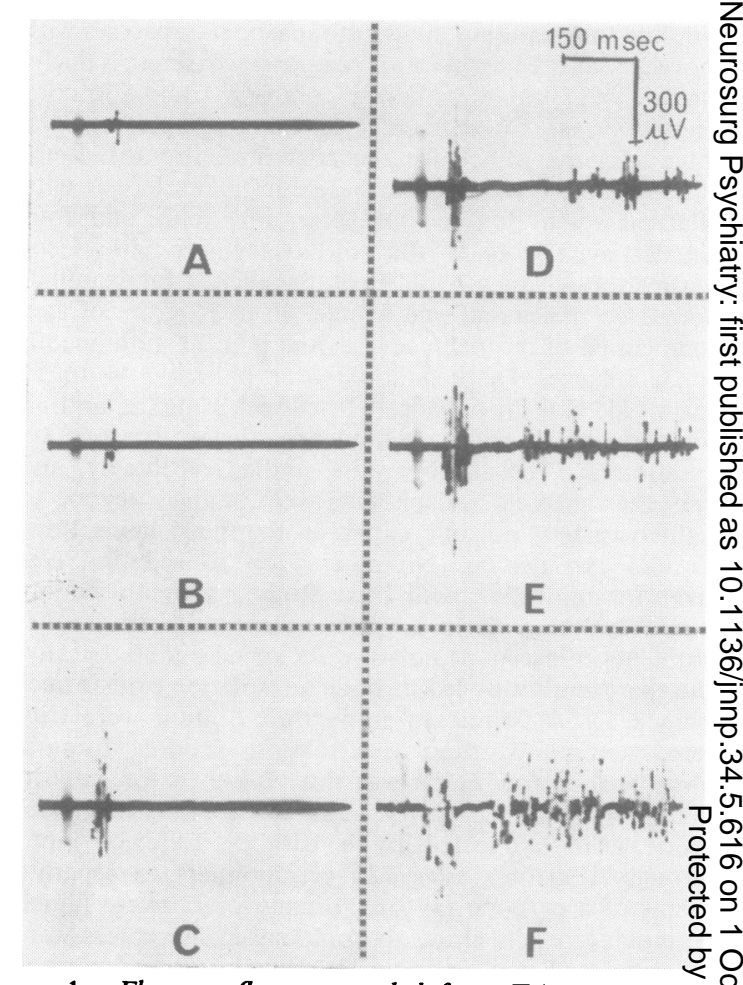

FIG. 1. Flexor reflexes recorded from $T A$ in a normgl subject. Stimulus trains, as noted in the text, applied to the sole of the ipsilateral foot. Stimulus voltage increased from (A) through (F), 15, 20, 25, 30, 35, and $40 \mathrm{~V}$. Calibrations $\frac{0}{+} \overrightarrow{+}$
150 msecs and $300 \mu \mathrm{V}$. See text for explanation.

stimulus intensities, it was often impossible to see two separate components (Fig. 2). The duration of the first, which initially may have been $5 \mathrm{msec}$, now was at least $60 \mathrm{msec}$ and merged with the second one $\overrightarrow{\vec{F}}$

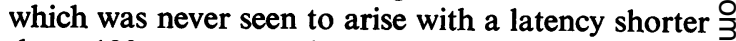
than 120 msec. Both components increased in $\vec{P}$ amplitude and duration as stimulus intensity was increased though the second, when present, was usually much larger than the first. Most often the first component appeared at a lower stimulus intensity than the second (Fig. 1). Furthermore, the first 3 component produced, at most, a brief small amplitude twitch, while the second was associated with $\frac{\text { 을 }}{3}$ gross movement and withdrawal of the limb (Fig. 3). Single stimuli were capable of producing both components in some subjects but a train of stimuli $\frac{D}{O}$ was more reliable in that it was adequate for the production of both components as well as being $\mathrm{N}$ supramaximal in all subjects. We have no data $N$ suggesting any qualitative difference in response to one stimulus as opposed to a train. 


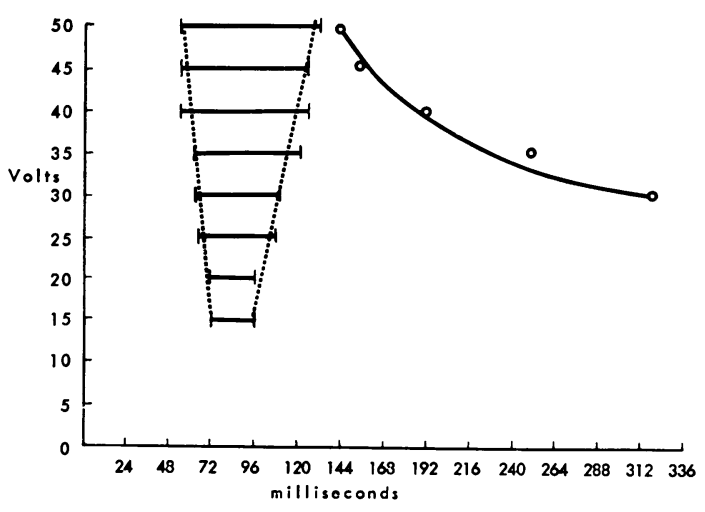

FIG. 2. Strength-latency curve for normal subject as in Fig. 1. Ordinate represents the strength in volts of the stimulus trains and the abscissa the latency and duration of the first and latency of the second component. Note that, as stimulus intensities are increased, the latency of the first component decreases while the duration increases. Ultimately, a second component appears and with stronger stimuli the latency of this decreases markedly.

Excitability curves plotted, using two equal sets of stimuli through the same electrodes at various intervals, always showed an initial phase of facilitation lasting two to four seconds after the first

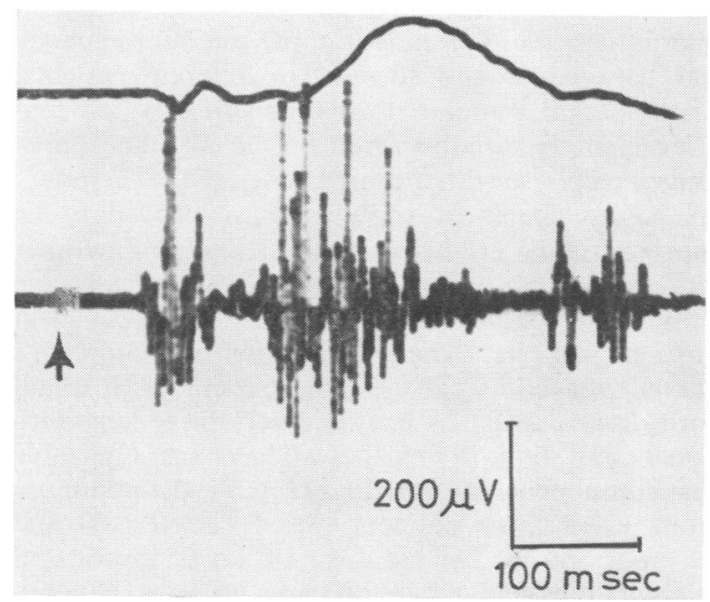

FIG. 3. A combined EMG and mechanogram of a typical flexor reflex. The lower line is the EMG recorded from $T A$ in response to a train of stimuli (arrow) applied to the sole of the foot, as noted in the text. The upper line is the mechanogram recorded from a silicone transducer applied to the dorsum of the foot, depicting movement at the ankle produced by the flexor reflex. Calibration for the EMG is $200 \mu \mathrm{V}$ and for both lines $100 \mathrm{msec}$. stimulus (Fig. 4). The reflex response to the second stimulus was then of much larger amplitude and duration. This phase was followed by a period of subnormality, lasting for five to 10 seconds, during which the response to the second stimulus was much smaller in both dimensions and often entirely absent. During the initial facilitatory phase the latency of the reflex from the second stimulus was always decreased compared with the first or 'conditioning' stimulus. Similarly, during the next or subnormal phase, the minimum latency of the second reflex was longer than that of the first. Thereafter, a phase of recovery was found so that two sets of stimuli separated by 60 seconds or more produced equal responses (Fig. 5). These curves characteristically were plotted using the same subject on a number of days, and their forms were remarkably similar.

Various facilitatory and inhibitory cutaneous areas for this particular flexor reflex were mapped out by scratching, pinching, rubbing, pricking, or pulling the hairs of different parts of the lower extremities and recording the effect on the latency and size of the control flexor reflex. Our results were

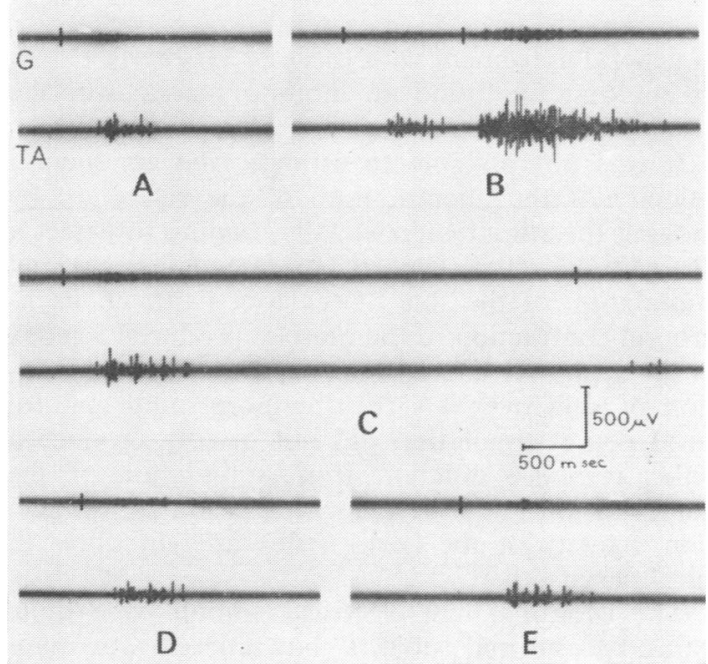

FIG. 4. Flexor reflex responses from a normal subject in response to identical stimulus trains, given at different intervals. The lower line depicts the EMG response in $T A$, and the upper the response recorded from gastrocnemius. Stimulus artefacts have been retouched on the upper lines. (A) is the control response, (B) the responses to two trains of stimuli given 1 sec apart, (C) the responses with the two trains given $4 \cdot 2 \mathrm{sec}$ apart, (D) the initial response, and (E) the second response with the trains separated by $45 \mathrm{sec}$. Calibration $500 \mathrm{msec}$ and $500 \mu \mathrm{V}$. Note that the second response is larger with a briefer latency in (B). It is much smaller in (C) and similar to the control in (E). 


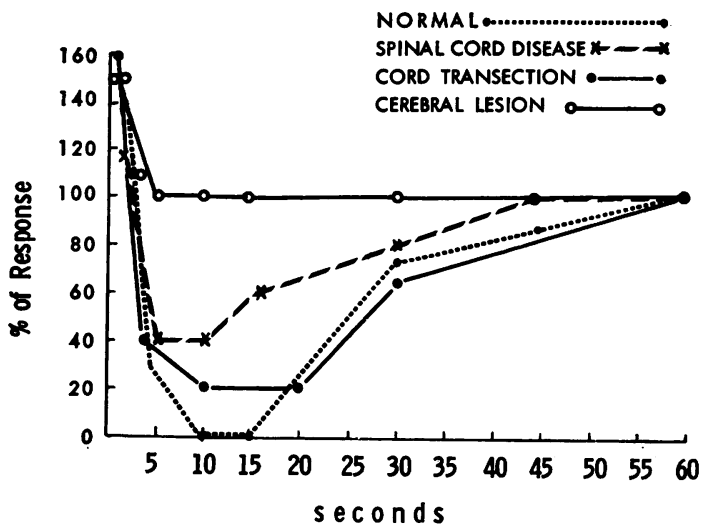

FIG. 5. Excitability curves from three patients and one normal subject, as noted. The curves are drawn from data such as those in Fig. 4. Ordinate represents the size of the second flexor reflex in arbitrary units, the initial or control reflex being $100 \%$. The abscissa represents the time interval in seconds between the two identical trains of stimuli. Note the initial phase of facilitation in all four records and the subsequent phase of depression in all except the patient with the cerebral lesion.

in general agreement with those of Hagbarth (1960) except that we found an inhibitory area over the dorsum of the foot.

It was not possible to estimate the conduction velocity of the afferent limb of the reflex arc by moving the stimulus proximally, hoping to detect a decrease in reflex latency. In fact, supramaximal stimulation of the sole of the foot (with no background contraction in the muscle) produced a reflex in TA at shorter latency than supramaximal stimulation of skin over TA. Furthermore, quite painful sural nerve stimulation did not usually produce a reflex response different from stimulation of the adjacent skin so we were unable to estimate conduction velocity of the flexor reflex afferent fibres in sural nerve either.

The supramaximal electrical stimuli were quite painful to normal subjects, but reflexes with minimum latencies could rarely be produced otherwise. In studies of this sort, the influence of 'cerebral' processes is very difficult to evaluate, whereas this is not a problem when studies are carried out with spinal man. In normal subjects apprehension or fear seemed mainly to decrease or inhibit the first component while the second remained either unchanged or increased in amplitude. The converse psychic state, 'habituation' or relaxation, had equally potent influences on flexor reflexes. As noted above, if an interval of 60 seconds elapsed between two equal stimulations, the responses produced were essentially $Z$ identical. If, however, stimulation was repeated indefinitely, even at intervals greater than 60 seconds, the reflex response would begin to diminish after four to six repetitions. In particular, the second 6 component would decrease in size and increase in latency. This effect, which is defined as habituation, can be reversed, in large part, by apprehension. When habituation has taken place, the reflex response to an identical stimulus will be increased $\Rightarrow$ if the patient expects the stimulus to be more painful $\stackrel{?}{\rightarrow}$ than usual or if he is more apprehensive in general. 을 Attempts were made to control these factors when- 흠 ever possible and, as noted, care was taken to give $\frac{\bar{\omega}}{\partial}$ no clue as to when stimulation was to take place. $\stackrel{\odot}{\Omega}$ Nevertheless, even when using a silent trigger for stimulation and a random stimulus schedule, control of all the supraspinal influences on these reflexes is $\odot$ far from perfect. For this reason, reflex latency $\vec{\omega}$ seems the most reproducible parameter rather than amplitude or duration, since these latter are particularly sensitive to apprehension or habituation.

PATIENTS WITH SPINAL CORD LESIONS All 14 patients of who had clinically severe, though incomplete, spinas $\frac{\sigma}{\sigma}$ cord lesions behaved in a remarkably consisteri fashion and will be described together. It was difficult to separate two reflex components in these $\vec{e}$ patients-the response with the lowest threshold always had a prolonged latency (120 to $400 \mathrm{mseg} \frac{0}{0}$ (Fig. 6). Increasing the stimulus strength decrease the latency and increased the size of the reflex; $\overrightarrow{0}$ high intensities of stimulation, the minimum latency $\vec{v}$ was between 60 and $80 \mathrm{msec}$ in different patients. The apparent stimulus thresholds varied widely and a large single stimulus often produced a large prolonged response with a short latency $(60$ to $80 \mathrm{msec})$. As before, trains of stimuli always proved to be supramaximal, whereas single stimuli sometimes did not. Excitability curves (Fig. 4), using the double stimulus technique, were essentially the same as in 3 normal subjects. The patients with Friedreich's ataxia behaved as did the other patients with spinal cord disease as far as having a very large late component, but differ in that they all have a recognizable first component. In particular (Fig. 7), the minimum latencies in these patients varied between 60 and 3 $80 \mathrm{msec}$ despite severe loss of large myelinated afferent fibres shown by sural nerve biopsies (Hughes, Brownell, and Hewer, 1968).

The patient whose cord was transected was studied from the stage of severe spinal shock (when on flexor responses were small and feeble and tendon jerks and $\mathrm{H}$ reflex absent) to a stage at which both $\sigma$ tendon jerks and flexor reflexes were moderately $N$ brisk. The size of the flexor reflexes increased drama- N 


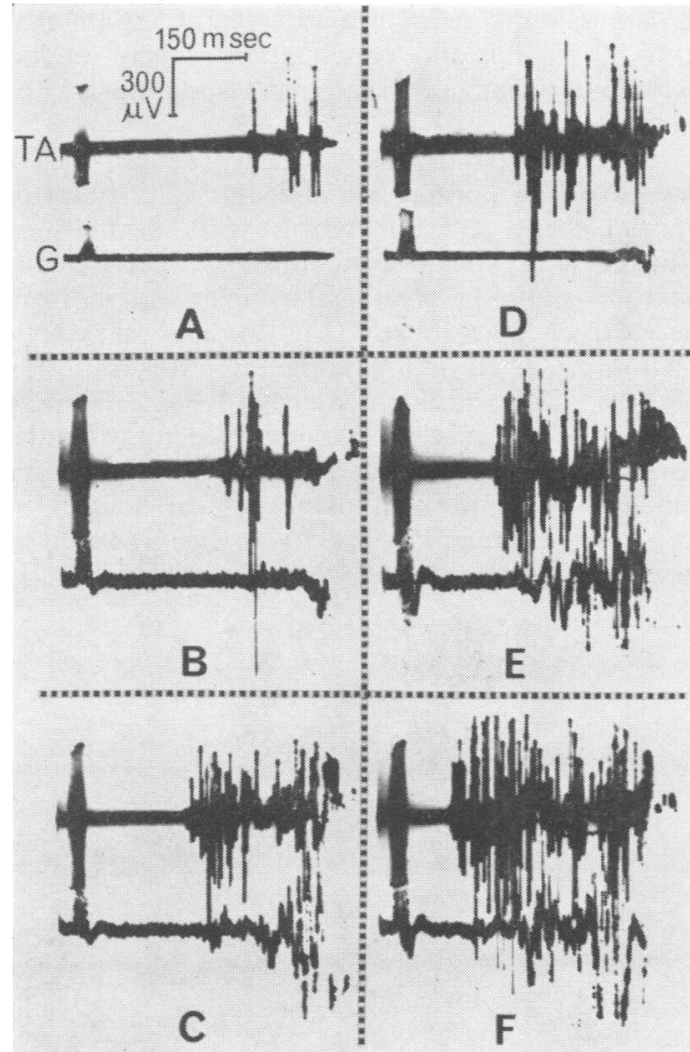

FIG. 6. Flexor reflex responses in a patient with spinal cord disease (multiple sclerosis). Contrast these with a similar experimental situation in a normal subject in Fig. 1 . The upper line in each represents the EMG recording from $T A$, and the lower line that from gastrocnemius. (A) to (F) represent increasing voltage of stimulus trains from 15 to $40 \mathrm{~V}$. Calibration-150 $\mathrm{msec}$ and $300 \mu \mathrm{V}$. For explanation, see text.

tically, and the apparent threshold decreased during that time, but their minimum latency did not vary. A single submaximal stimulus of 10 to $15 \mathrm{~V}$ and $0.1 \mathrm{msec}$ duration gave rise, regularly, to a small flexor reflex with latency about $400 \mathrm{msec}$. The latency decreased as larger stimuli were given but at moderate intensities, when the latency was still 120 to $240 \mathrm{msec}$, a new component was seen with latency 50 to $65 \mathrm{msec}$ (Fig. 8). This earliest reflex was similar to, though much smaller and more easily fatigued than, the normal first component. Even stronger stimuli produced prolonged reflexes with latency 50 to $65 \mathrm{msec}$. Single stimuli all had identical efficacy with those in the other 14 patients with spinal cord disease. The flexor reflexes in this

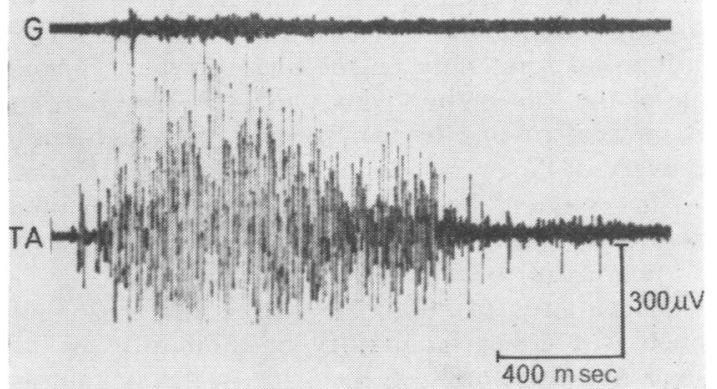

FIG. 7. Flexor reflex response recorded from a patient with long-standing Friedreich's ataxia. The lower line represents the EMG from $T A$, and the upper line that from gastrocnemius. The stimulus train itself triggered the sweep, and the stimulus artefact is not well seen. Calibration is $400 \mathrm{msec}$ and $300 \mu \mathrm{V}$. Note the brief latency and separation of the reflex into a small initial and large second component.

patient, however, particularly those evoked early in his recovery from spinal shock, were very sensitive to repetition. The reflex response very rapidly decreased and often disappeared as the number of stimulus trains exceeded three or four, even with a 60 second interval between each one. The lability of flexor reflexes in this regard varied from day to day for reasons that are unclear to us, and recovery from this 'exhaustion' or 'depression' required five to 10

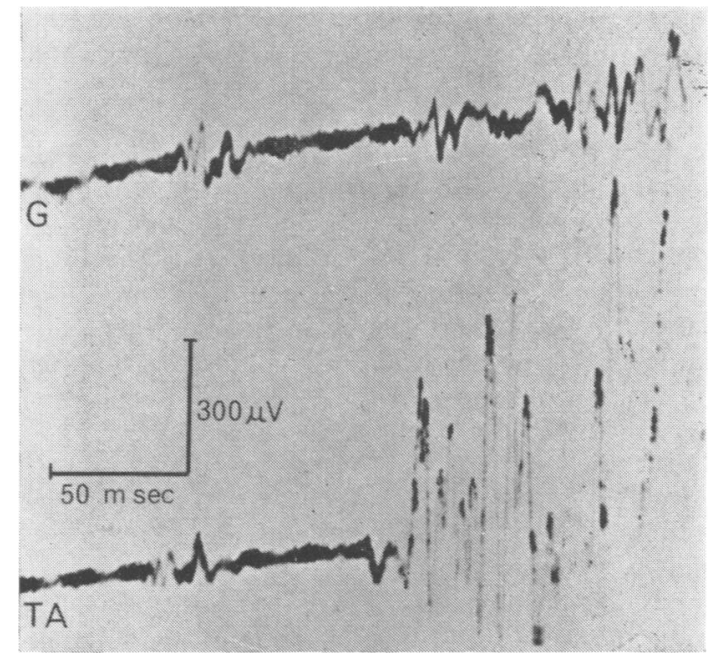

FIG. 8. Two components of the flexor reflex recorded from $T A$ in a patient whose spinal cord was transected. Stimulus as in Fig. 7. Calibration is $50 \mathrm{msec}$ and $300 \mu \mathrm{V}$ For details, see text. 
minutes of rest. During this phase of depression, it was possible to elicit reflexes more nearly normal in size by combining the electrical stimulus with mechanical scratching or rubbing elsewhere on the sole of the foot or by giving two or three stimuli at an interval of one to two seconds. Studies of the recovery of flexor reflexes over long periods of time in this patient prompted us to investigate the excitability cycles of flexor reflexes in all our subjects at intervals of seconds and minutes.

The patterns of cutaneous areas, stimulation of which was either facilitatory or inhibitory to the flexor reflexes, were also the same in this patient as in the other patients with spinal cord disease. This was particularly easy to demonstrate early in the recovery from spinal shock at the stage in which flexor reflexes had not yet become elicitable by the slightest stimuli and before spontaneous flexor spasms began to occur.

In general, in all patients with spinal cord lesions these patterns differ from those in normal subjects primarily in that flexor reflexes tend to predominate and can be produced in some instances from mechanical stimulation of any cutaneous area of the leg. If, however, the mechanical stimulus is adjusted so as to be subthreshold for the production of such reflexes, it will then condition an electrically elicited flexor reflex in much the same way as it would in normal subjects. Supramaximal stimuli were not particularly painful in these patients and, in fact, did not always prevent them from falling asleep during the studies. Similarly, variations in the level of apprehension were less of a problem than in normal subjects. Apprehension or conscious pain certainly did not appear to influence the reflexes in the isolated spinal cord.

PATIENTS WITH DISCRETE CEREBRAL LESIONS Preliminary studies in five patients with reasonably discrete lesions of the cerebral hemispheres are presented as a contrast to the foregoing. The situation with these lesions is much more complicated than with spinal cord disease because different sites and severity of cerebral lesion produce different clinical syndromes involving the limbs, and further study in this regard is presently under way.

In these patients, a discrete component with latency 50 to $80 \mathrm{msec}$ is rarely seen, and the largest painful trains of stimuli produced, in only three of the five, a prolonged reflex that began with approximately $80 \mathrm{msec}$ latency. Two large trains delivered within three or four seconds, on the other hand, always produced, in response to the second at least, a large reflex with 50 to $80 \mathrm{msec}$ latency. However, the remainder of the excitability curve (Fig. 4) differs from those described previously insofar as no depression of reflex size was seen at any interval $\underset{\mathbb{D}}{Z}$ up to 60 seconds and, concomitantly, there was no increase in latency of the second response at any of these intervals.

Furthermore, though the reflex produced in TA 0 was usually of normal size and duration, it was of unusual shape and, in contrast with normals or patients with spinal cord disease, simultaneous recording showed a great deal of reflex activity from $\$ the gastrocnemius muscle (Fig. 9). The reflex con- $\overrightarrow{\vec{A}}$ traction produced in TA by the painful stimuli was $\frac{?}{0}$ followed, at an additional latency of 60 to $150 \mathrm{msec}$ 등 in different subjects, by a burst of activity in the $\frac{\bar{C}}{\bar{D}}$ gastrocnemius muscle. Each of these bursts, in turn, $\vec{\varnothing}$ coincided with transient depression of activity in $\varrho$ TA, and alternating clonic activity was produced in these two antagonistic muscles.

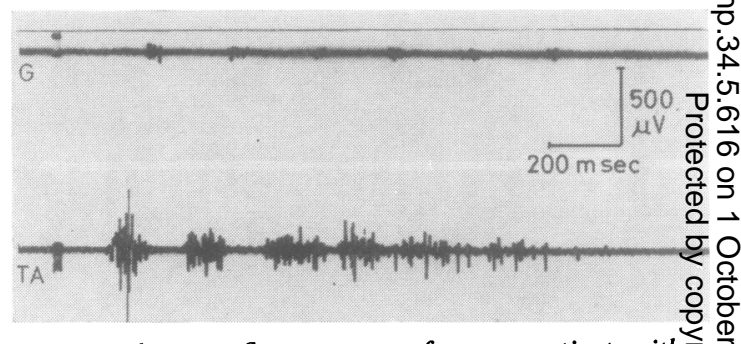

FIG. 9. Flexor reflex response from a patient with discrete parietal lobe lesion, contralateral to the leg beim studied. The lower line is the EMG from $T A$, and the upper $\rightarrow$ line EMG from gastrocnemius. Calibration is 200 msec $_{\square}$ and $500 \mu \mathrm{V}$. Note the long latency of the flexor reflex and the alternating activity in the flexor and extensor muscle groups. See the text for further explanation.

PATIENTS WITH PARKINSON'S DISEASE Studies of flexor reflexes in these five patients are presented to illustrate other changes that may take place in such reflexes under pathological conditions. The most significant difference between the reflex response ino normal subjects and patients with Parkinsonism was that, in the latter, the first component had a relatively low threshold and could be evoked regularlyo by either a single stimulus or trains of stimuli. Indeed, this is the only neurological disorder we을 have studied in which the stimulus threshold for $>$ evoking the first component of the flexor reflex is lowered. On the other hand, the latencies are normaln (55 to $65 \mathrm{msec}$ for the first and 135 to $190 \mathrm{msec}$ for the second component, depending on strength of stimulation). 


\section{DISCUSSION}

The importance of methodology in studies of this type cannot be overemphasized. Because of the many independent variables in such a study, the results, though reproducible within any system, are difficult to compare with those of others unless the techniques are also comparable. Interpretations and conclusions which may follow from the results are highly dependent upon details of the experimental situation. For example, it is in certain circumstances, possible to obtain minimal flexor reflex latencies with afferent stimulation which is, in fact, submaximal by our criteria-for example, if such stimulation is superimposed upon a background of voluntary contraction in TA. Utilizing this technique, however, reflex latencies are the same after stimulation of many different cutaneous areas over the lower extremity and foot. It, therefore, becomes impossible to evaluate gradations in the relative effectiveness of afferent stimulation from different cutaneous areas ('local sign') except by very crude estimation of whether an early latency reflex is present or absent. Furthermore, different patterns of reflex activity are found as the normal subject changes position and posture from lying supine to being seated or standing. With the subject standing, activity and latencies in TA vary greatly depending on whether he leans very slightly forward or backward. These changes are mediated, in part, through differences in background contraction or 'central excitatory state' in the flexor motor neurone pool. The employment of voluntary contraction as a technique also is obviously impossible in spinal man. We have, therefore, chosen to record activity with the motor neurone pools being free of voluntary or postural activity as far as that is possible.

One must also remember the other posturally dependent inputs, for example, from labyrinthine and particularly from cutaneous receptors. 'Local sign' is a very important aspect of flexor reflexes as measured by changes in the patterns of muscle activity produced by small changes in stimulus site. We were able to confirm many of the findings of Hagbarth (1960) in regard to the effect of changes in the site of cutaneous stimulation as measured by the flexor reflex in TA. For this reason, it would seem simpler and more natural to stimulate a small area of skin by surface or in-dwelling electrodes rather than to stimulate the fibres even in a 'cutaneous' nerve. These fibres come from a large area of skin as well as from receptors in joint capsules, deep fascia, and other deep connective tissue. If mixed nerves, such as the posterior tibial, are stimulated at the ankle through the skin, one would stimulate endings in the skin overlying the nerve as well as the largest lowest threshold fibres in the nerve itself. These latter obviously come from a number of different areas of skin over the sole of the foot and from muscles, joints, and so forth throughout the foot. Such varied input to the spinal cord may, in fact, tend to produce competitive reflex responses. The intradermal needles probably excite terminal branches of cutaneous afferent fibres and not nerve endings themselves. These terminal branches also come from nearby fields, but the area of skin from which these endings come would be much less than if the entire nerve trunk were stimulated. Two generalizations have emerged from studies of stimulation via intradermal needles in the glabrous skin of the monkey (Mountcastle and Powell, 1959; Powell and Mountcastle, 1959). On the one hand, Mountcastle and Powell found that stimulus durations of the order of $0.75 \mathrm{msec}$ were required to produce more than one impulse per stimulus in any primary fibre of the dorsal column. With impulse durations of $0.1 \mathrm{msec}$, no more than one impulse per stimulus was recorded from any single fibre, and changes in reflexes produced by increasing or decreasing the stimulus strengths are due to increase or decrease in the number of active fibres rather than in the number of impulses per stimulus in any given fibre. The other point is that electrical stimulation of the skin is a convenient way of confirming the cutaneous (as opposed to deep) origin of the excitatory afferent impulses responsible for flexor reflexes. Though electrical stimulation of this sort produces an abnormally synchronized afferent volley which would be a very unusual result of normal tactile stimulation, no qualitative difference in the nature of responses, either in the primary afferent neurone or in cortical neurones, was found when this form of electrical stimulation was compared with brief mechanical or tactile stimulus to the skin. Furthermore, the afferent volley is, in all likelihood less synchronous with this intracutaneous form of stimulation than with stimulation of the cutaneous nerves themselves more proximally.

Because of the marked changes produced in flexor reflexes by differences in site of stimulus, we were, as noted above, unable to confirm Hagbarth's (1960) studies in which he attempted to evaluate the conduction velocity of the flexor reflex afferent fibres by stimulating the leg at two sites, one more proximal than the other. We were, however, able to utilize the striking peripheral neuropathy found in Friedreich's ataxia to give us some information as to the afferent nerve fibres responsible for flexor reflexes. It has been shown by Hughes et al. (1968), Dyck, Gutrecht, Bastron, Karnes, and Dale (1968), Dyck and Lambert (1968), and MacLeod (1969) that the largest myelinated fibres in cutaneous nerves from 
patients with advanced Friedreich's ataxia are markedly reduced in number or absent. These patients in our study had first and second components with normal minimal latencies. It would, therefore, appear that the largest myelinated afferent fibres are not necessary for the production of flexor reflex responses with latencies of 50 to $80 \mathrm{msec}$. This agrees with the original observation of Kugelberg (1948) that the smaller myelinated fibres (which are preserved in Friedreich's ataxia) may carry the afferent activity responsible for these reflexes. That fibres such as these with conduction velocities of 35 to 40 metres/sec are the afferent fibres responsible for human flexor reflexes has recently been confirmed by one of us (Shahani, 1970). It is unlikely that 'unmyelinated' or C fibres would be responsible for these short latency reflexes because conduction in them is far too slow to allow for these minimal latencies.

The patients with Friedreich's ataxia are interesting in another respect. They have degeneration of the corticospinal tracts in the cord, positive Babinski signs, very easily elicited flexor reflexes, and, as a rule, spontaneous flexor spasms, though they tend to be mild. Their flexor reflex behaviour would appear to differ, however, from patients who have primary spinal cord disease of traumatic or demyelinative type. A first component is routinely seen in patients with Friedreich's ataxia but infrequently in those patients with easily elicited flexor reflexes because of lesions which primarily affect the spinal cord.

These flexor reflexes are clearly not myotatic or proprioceptive and neither are they similar to Sherrington's scratch reflex. The latter is not elicitable by a single stimulus, whereas in both cats and man flexor reflexes can be so produced. Shimamura and Livingstone (1963) have described a reflex system in the decerebrate cat in which one stimulus evokes two responses, one a spinal reflex and the other a 'spinobulbospinal' (SBS) reflex in which the medullary reticular formation is the reflex centre. In a study in man, a reflex has been described in TA which occurred 70 to $80 \mathrm{msec}$ after stimulation of the tibial nerve in the popliteal fossa, and it was considered to be a SBS reflex (Shimamura, Mori, Matsuchima, and Fujimori, 1964). Since we found two discrete components in the patient with a spinal transection, it seems unlikely that either of these represents a SBS reflex. Furthermore, the afferent fibres to the medullary reflex centre of the SBS reflex may be large myelinated cutaneous fibres, whereas our evidence suggests that small myelinated fibres are the afferents responsible for both components of the flexor reflex.

The physiological significance of these two separ- ate components, described by Tureen (1941), Lloyd $\mathcal{Q}$ (1943), Kugelberg (1948), Brooks and Fuortes (1952), Pedersen (1954), Henneman (1957), and क Hagbarth and Finer (1963) is far from evident. It is clear that both arise from the same muscle, and 0 other experiments (Shahani, 1968, 1969) suggest that they often arise from the same motor units, though $\stackrel{2}{2}$. further study of single motor unit behaviour will be interesting in this regard. Because either first or $:$ second component can be seen without the other in $\overrightarrow{\vec{F}}$ certain circumstances with identical stimulation, it is $\frac{\overrightarrow{0}}{0}$ unlikely that these two components arise from $\frac{C}{0}$ activity in two separate groups of afferent fibres. The second, which may occur alone, is also not $\frac{\mathbb{\Phi}}{\mathrm{O}}$ caused by the first. Because occasional isometric 2 recordings also show both components, it is unlikely क that these are two portions of the same reflex with $\overrightarrow{0}$ an intervening silent period, produced, perhaps, by $\vec{\overrightarrow{ }}$ stretching of the extensor muscles. Furthermore, the $\vec{\omega}$ two components are found in patients with Friedreich's ataxia in which there are neither tendon jerks nor tonic stretch reflexes. We cannot deny that $\omega$ recurrent inhibition consequent on the initial some- $\vec{c}$ what synchronous firing of the flexor motor neurones os may account for a fraction of the separation in $\frac{\overrightarrow{6}}{\sigma}$ two components, especially with submaximal 윽 stimuli, but further investigations of this problem are needed. Though these two components a obviously related, they do behave in many ways if $\frac{O}{0}$ an opposite manner. For example, the first com ponent may be present with the second reduced of: absent, either at levels of stimulus which are sut maximal or with habituation, whereas the second.component may be present without the first during apprehension or sleep in normal subjects (Shahani, $1968,1969)$ and in various neurological disorders. Furthermore, they appear to differ functionally, and while the second component clearly is associated $\stackrel{\circ}{\square}$ with most of the movement produced by these $\underset{\vec{F}}{\overrightarrow{2}}$ reflexes, the significance of the first component is, at $\frac{0}{3}$ most, conjectural.

Clues to the possible significance of the first component may be obtained by consideration of the circumstances in which it is not found. For example, when it is absent as in sleep or in the presence of spinal cord disease, the flexor withdrawal responses 3 - that is, the second component-are highly exaggerated and stereotyped. If the latter observations $\frac{\circ}{3}$ are causally related to the former, it might suggest that the first component regulates, in some inhibitory fashion, the movement produced by the second. In $\rightarrow$ a recent study of monoaminergic systems in the cat, Lundberg (1966) describes short latency flexor reflex $N$ pathways which have strong inhibitory effects on longer latency flexor reflex responses. These latter, $\mathcal{O}$ when released by the administration of L-dopa, are $\omega$ 
prolonged and intense. It is noteworthy that the converse is seen in Parkinsonism, a pathological condition associated with depletions of monoamines in the central nervous system (Barbeau, 1962, Hornykiewicz, 1966) and some of the symptoms of which are relieved by the ingestion of L-dopa (Delwaide, Schwab, and Young, 1971). We have found that patients with Parkinsonism have a first flexor reflex component with unusually low threshold, and they have no tendency to flexor spasms despite a predominantly flexed posture. Further studies of these reflexes and the changes in them produced by pharmacological alteration in monoaminergic pathways should prove fruitful.

Previous studies (Pedersen, 1954; Hagbarth, 1960; Kugelberg et al., 1960; Dimitrijević and Nathan, 1968) have usually focused upon the reflexes with minimal latency. This emphasis on the initial component was because those parts of the response which appear with longer latencies than $120 \mathrm{msec}$ were considered by Hagbarth and Finer (1963), for example, to be a 'cerebral' reflex. Studies of spinal man are particularly significant in this regard. The second component in this patient is not obviously different from that in other subjects studied, and since it clearly is spinal in origin, the cerebral aspects of the second component in other subjects may be less than formerly thought. In addition, a first component was seen in spinal man and, in this regard, is different from those other patients with chronic spinal cord lesions. Since the spinal man was studied early after recovery from spinal shock and the other patients all much later in the course of their spinal cord disease, this may account for the discrepancy, though there may also be other physiological differences. As his flexor reflexes became increasingly labile, the first component became smaller and more difficult to obtain. Though the first component is difficult to record in patients with primary spinal cord disease or those with cerebral lesions, this does not imply that the mechanism responsible for its absence is the same in both instances. As an illustration of this difference, it is quite easy to produce a flexor reflex with minimum latency in patients with spinal cord disease even though the first component never appears in isolation. On the other hand, it was difficult to get short latency flexor reflexes in the patients whom we studied with cerebral lesions-it was possible to produce them only by arranging for the stimulus to fall into the facilitatory period a few seconds after a previous stimulus. This effect, as well as the early phase of the excitability curve in general, where the second response is much larger than the first, is presumably an example of temporal summation in which the subliminal fringe of the flexor moto- neurone pool is activated. This also accounts for the observation that, in the next phase, the depression can be at least partially overcome by stimulating yet again within a few seconds of the second stimulus, the response to the third stimulus being larger and of briefer latency. This depression can also be partially overcome by spatial summation-that is, by simultaneous stimulation (mechanical or electrical) of other skin areas.

The patients with primary spinal cord lesions behave in a remarkably homogeneous fashion and differ from normal principally in that thresholds for the production of flexor reflexes are often lower and the reflex itself of much larger amplitude and duration. Despite this, it is necessary to use stimuli of much greater intensity in these people to produce reflexes with minimal latencies as brief as normal. Because the similarities between this group and the findings in the patient whose spinal cord was transected are so striking and because in this patient we may ascribe all reflex findings to purely spinal mechanisms, it is worth stressing the conclusions that may be drawn from the study of spinal man. Despite the preponderance and lability of flexor reflexes even early in the course of such patients, evidence of the mechanisms of 'local sign' initially described by Hagbarth $(1952,1960)$ is to be found if the mechanical stimuli are carefully adjusted to be subthreshold for the production of flexor reflexes. This differs from the statements of Dimitrijević and Nathan (1968), but one must be careful to relate the findings in spinal man to the stage these patients have reached following cord transection. It is, for instance, much easier to demonstrate the effect of stimulation of different cutaneous areas early in the course of such patients, shortly after recovery from spinal shock, than it is later when the flexor reflexes have become extremely labile. Nevertheless, it is important to note that these physiological mechanisms, even if demonstrable primarily early in the patient's course, nonetheless must depend entirely upon spinal mechanisms for their function.

Though our studies were not designed to test reciprocal inhibition, we can examine the relationship between activity recorded from TA and gastrocnemius muscles. This relationship in patients with spinal cord disease contrasts strikingly with that in patients who had cerebral lesions. In the latter (Fig. 9), activity alternating between flexor and extensor antagonists is clearly seen. This differs from ordinary clonus in which periodic activity is found in triceps surae but no activity in TA (Cook, 1967). However, ordinary clonus is elicited by sustained stretch of triceps surae, whereas in our studies, the primary afferent input is in 'flexor reflex afferents'. This produces reflex contraction of TA 
which stretches triceps surae and may thereby produce the reflex recorded from the gastrocnemius muscle. A background central excitatory state apparently persists in interneurones leading to the flexor motoneurone pool, and after gastrocnemius motoneurones fire, activity is seen again in TA. This alternate firing and silence in these antagonistic muscles may well represent reciprocal inhibition, that is reflex coordination at the spinal level in patients with cerebral lesions. On the other hand, the human spinal cord, below a severe lesion or complete transection, sustains activity in both flexor and extensor motoneurones at the same time, though our stimulus produced earlier contraction in TA, which may then stretch triceps surae and produce a stretch reflex therein. The difference between these two types of patients is that, in those with spinal cord disease, reflex activity of flexor motoneurones does not appear to be associated with depression of activity in extensors or vice versa, the situation seen with the clonic activity described above. That is not to suggest that all segmental reflex coordination is in abeyance in the isolated human spinal cord. Clonus produced by sustained dorsiflexion of the foot in paraplegics can be overcome by pinch, hyperflexion, or other 'painful' stimulation to the foot.

To return to spinal man, it was soon found that the latency and size of the flexor reflex produced electrically were very dependent upon any evoked or spontaneous flexor activity in the extremity immediately preceding the reflex which was being tested. Repetitive stimulation soon results in a much smaller reflex, and whether this depression of reflex activity is the result of transmitter depletion, fatigue in a more general way, inhibition, or 'habituation' remains to be seen. The pain produced by the second train of stimuli is, in the normal subject, no less than that produced by the first even if the trains are separated by four or five seconds so there is no reflex response to the second one. This is also true of trains repeated more often and signifies that, as one would expect, there is no fatigue or habituation of the peripheral afferent system, nor indeed any of the central afferent system, responsible for pain. If the same afferents produce pain and flexor reflexes, the cause of the phase of depression of the latter must lie in the flexor motor neurones or in the interneurones leading to them from collaterals of the ascending afferent fibres. One must remember, however, that activity in the afferent fibres responsible for pain may not always be necessary for the production of flexor reflexes. In normal man and particularly in those patients with spinal cord disease, flexor reflexes may be produced by light touch and other apparently non-noxious cutaneous stimuli.
These findings of depression of reflex response with repetition resulted in our studying the excitability curve using two sets of stimuli at various $\stackrel{\infty}{\subseteq}$ intervals. Certainly, very long-lasting effects-that $\widehat{C}$ is, many seconds-are produced by these reflexes in $D$ terms of the period of depression which is found following one set of stimuli or one spontaneous $\frac{\vec{N}}{\bar{g}}$ flexor spasm. Furthermore, it is quite simple by our technique to record flexor activity from the isolated human spinal cord with an extremely long latency- $\overrightarrow{\overrightarrow{\vec{S}}}$ for example, $400 \mathrm{msec}$-after a single low intensity $\overrightarrow{0}$ intradermal stimulus which is $0.1 \mathrm{msec}$ in duration. 흘 We assume that such a stimulus produces only one $\overline{\bar{c}}$. impulse per fibre stimulated (Mountcastle and $\widehat{\varnothing}$ Powell, 1959) and that 'unmyelinated', very slowly conducting fibres are not activated. It follows, there- $\infty$ fore, that a single afferent volley can set up activity $\vec{\circ}$ in the cord which produces flexor reflex discharge $\overrightarrow{\vec{H}}$ hundreds of milliseconds later, and that this repe- $\omega$ titive firing of motoneurones is not dependent upon repetitive input in any first order afferent fibre. It seems unlikely that very long chains of interneurones $\omega$ are responsible for such extremely delayed reflex activity, and studies of the spinal mechanisnof involved have only begun in the animal laborato (Andén, Jukes, Lundberg, and Vyklicky, 1964. 을 Landau and Clare (1959) have shown that fibrilla-tions may be recorded from the paralysed muscles? of patients with spinal cord transection very eargy $\frac{?}{0}$ in their course. We did not evaluate the spinal min for possible fibrillations, but no denervation evident clinically, and reflex activity eventualy became very active indeed. It is doubtful that ${ }^{-}$ minimal denervation, which may have occurred, would be responsible for the reflex activity seen with very prolonged latency, for the two components or for the relatively normal integration from the cutaneous areas in this patient.

The excitability curve is much the same in patients $\Rightarrow$ with spinal lesions as it is in normal man, and it 3 may well be that the delayed depression seen in? normal subjects also is on a spinal basis. This is in contrast with the patients with cerebral lesions. in whom no such phase of depression is seen.? This absence of depression would tend to suggest: that transmitter depletion, for instance, or fatigue in some general sense is not reponsible for the depression seen in normal or spinal man. Similar conclusions may be drawn from the presence of reversibility of habituation noted above in normal or spinal man.

These alterations in reflex patterns associated with lesions at different levels of the neuraxis are strikings and lead us to question the utility of a global conceph such as 'spasticity'. Certain clear-cut physiologica? differences have been documented in the reflex 
behaviour underlying 'spasticity' due to spinal cord lesions when compared with that resulting from hemispheral lesions. There obviously are different types of 'spasticity'-qualitatively as well as quantitatively-affecting different reflex systems. Those discussing 'spasticity', its pathophysiology or treatment, should be specific as to which type is under consideration. As further studies are carried out in patients with well-documented small lesions or clear-cut syndromes, better understanding may be reached of normal motor mechanisms and their perversions with disease, such as 'spasticity' and 'rigidity'. It may ultimately become possible to use tests similar to these as aids to the localization and characterization of lesions in the nervous system on a functional basis.

We wish to thank Professor W. Ritchie Russell for his constant encouragement and advice and Dr. G. Rushworth for the use of laboratory facilities. The preparation of this manuscript was underwritten by a grant from Mrs. W. B. Lloyd.

\section{REFERENCES}

Andén, N. E., Jukes, M. G. M., Lundberg, A., and Vyklický, L. (1964). A new spinal flexor reflex. Nature (Lond.), 202, 1344-1345.

Barbeau, A. (1962). The pathogenesis of Parkinson's disease: a new hypothesis. Canad. med. Ass. J., 87, 802-807.

Brooks, C. McC., and Fuortes, M. G. F. (1952). Electrical correlates of the spinal flexor reflex. Brain, 75, 91-95.

Cook, W. A., Jr (1967) Antagonistic muscles in the production of clonus in man. Neurology (Minneap.), 17, 779-781.

Copland, J. G., and Davies, C. T. M. (1964). A simple clinical skin electrode. Lancet, $1,416$.

Creed, R. S., Denny-Brown, D., Eccles, J. C., Liddell, E. G. T., and Sherrington, C. S. (1932). Reflex Activity of the Spinal Cord. Clarendon Press: Oxford.

Delwaide, P. J., Schwab, R. S., and Young, R. R. (1971). Polysynaptic spinal reflexes in Parkinson's disease: effects of L-dopa treatment. Neurology (Minneap.), (in press).

Dimitrijević, M. R., and Nathan, P. W. (1968). Studies of spasticity in man. 3. Analysis of reflex activity evoked by noxious cutaneous stimulation. Brain, 91, 349-368.

Dyck, P. J., Gutrecht, J. A., Bastron, J. A., Karnes, W. E., and Dale, A. J. D. (1968). Histologic and teased-fiber measurements of sural nerve in disorders of lower motor and primary sensory neurons. Mayo Clin. Proc., 43, 81123.

Dyck, P. J., and Lambert, E. H. (1968). Lower motor and primary sensory neuron disease with peroneal muscular atrophy. II. Neurologic, genetic, and electrophysiologic findings in various neuronal degenerations. Arch. Neurol. (Chic.), 18, 619-625.

Hagbarth, K.-E. (1952). Excitatory and inhibitory skin areas for flexor and extensor motoneurones. Acta. physiol. scand., 26 (Suppl. 94), $58 \mathrm{pp}$.

Hagbarth, K. E. (1960). Spinal withdrawal reflexes in the human lower limbs. J. Neurol. Neurosurg. Psychiat., 23, 222-227.
Hagbarth, K.-E., and Finer, B. L. (1963). The plasticity of human withdrawal reflexes to noxious skin stimuli in lower limbs. Progr. Brain Res., 1, 65-81.

Henneman, E. (1957). Relation between size of neurons and their susceptibility to discharge. Science, 126, 1345-1347.

Hornykiewicz, O. (1966). Dopamine (3-hydroxytyramine) and brain function. Pharmacol. Rev., 18, 925-964.

Hughes, J. T., Brownell, B., and Hewer, R. L. (1968). The peripheral sensory pathway in Friedreich's ataxia. Brain, 91, 803-818.

Kugelberg, E. (1948). Demonstration of A and C fibre components in the Babinski plantar response and the pathological flexion reflex. Brain, 71, 304-319.

Kugelberg, E., Eklund, K., and Grimby, L. (1960). An electromyographic study of the nociceptive reflexes of the lower limb. Mechanism of the plantar responses. Brain, 83, 394-410.

Landau, W. M., and Clare, M. H. (1959). The plantar reflex in man, with special reference to some conditions where the extensor response is unexpectedly absent. Brain, 82, 321-355.

Lloyd, D. P. C. (1943). Neuron patterns controlling transmission of ipsilateral hind limb reflexes in cat. $J$. Neurophysiol., 6, 293-315.

Lundberg, A. (1966). Integration in the reflex pathway. In Nobel Symposium. I. Muscular Afferents and Motor Control, pp. 275-305. Edited by R. Granit. Wiley: New York.

McLeod, J. G. (1969). Electrophysiological and histological studies in patients with Friedreich's ataxia. (Abstract.) Electroenceph. clin. Neurophysiol., 27, 723.

Mountcastle, V. B., and Powell, T. P. S. (1959). Neural mechanisms subserving cutaneous sensibility, with special reference to the role of afferent inhibition in sensory perception and discrimination. Bull. J. Hopk. Hosp., 105, 201-232.

Pedersen, E. (1954). Studies on the central pathway of the flexion reflex in man and animal and changes in the reflex threshold and the circulation after spinal transection. Acta psychiat. neurol. scand., Suppl. $88 ; 81$.

Powell, T. P. S., and Mountcastle, V. B. (1959). Some aspects of the functional organization of the cortex of the postcentral gyrus of the monkey: a correlation of findings obtained in a single unit analysis with cytoarchitecture. Bull. J. Hopk. Hosp., 105, 133-162.

Shahani, B. (1968). Effects of sleep on human reflexes with a double component. J. Neurol. Neurosurg. Psychiat., 31, 574-579.

Shahani, B. (1969). A Study in Human Reflexes. Ph.D. Thesis. Oxford University: Oxford.

Shahani, B. (1970). Flexor reflex afferent nerve fibres in man. J. Neurol. Neurosurg. Psychiat., 33, 786-791.

Shahani, B., and Young, R. R. (1969). Normal human flexor reflexes. (Abstract.) Electroenceph. clin. Neurophysiol., 27, 725.

Shahani, B., and Young, R. R. (1971). Flexor spasms in man. (In preparation.)

Sherrington, C. S. (1910). Flexion-reflex of the limb, crossed extension-reflex and reflex stepping and standing. $J$. Physiol. (Lond.), 40, 28-121.

Shimamura, M., and Livingstone, R. B. (1963). Longitudinal conduction systems serving spinal and brain-stem coordination. J. Neurophysiol., 26, 258-272.

Shimamura, M., Mori, S., Matsushima, S., and Fujimori, B. (1964). On the spino-bulbo-spinal reflex in dogs, monkeys and man. Jap. J. Physiol., 14, 411-421.

Tureen, L. L. (1941). Form of the reflex response in relation to the pattern of afferent stimulation. Proc. Soc. exp. Biol. $N . Y ., 46,543-544$. 